\title{
Erythroid-predominant myelodysplastic syndromes: enumeration of blasts from nonerythroid rather than total marrow cells provides superior risk stratification
}

\author{
Sa A Wang ${ }^{1,2}$, Guilin Tang ${ }^{1}$, Oluwole Fadare ${ }^{3}$, Suyang Hao ${ }^{1}$, Azra Raza ${ }^{4}$, Bruce A Woda ${ }^{1}$ \\ and Robert P Hasserjian ${ }^{5}$ \\ ${ }^{1}$ Department of Pathology, UMass Memorial Medical Center, University of Massachusetts, Worcester, MA, \\ USA; ${ }^{2}$ Department of Hematopathology, University of Texas MD Anderson Cancer Center, Houston, TX, USA; \\ ${ }^{3}$ Department of Pathology, Wilford Hall Medical Center, Lackland AFB, San Antonio, TX, USA; \\ ${ }^{4}$ Myelodysplastic Syndrome Program, St. Vincent's Comprehensive Cancer Center, New York, NY, USA \\ and ${ }^{5}$ Department of Pathology, Harvard Medical School, Massachusetts General Hospital, Boston, MA, USA
}

\begin{abstract}
In the FAB (French-American-British) and WHO (World Heath Organization) classifications, the blasts in erythroleukemia (M6a) are enumerated from the marrow nonerythroid rather than the total-nucleated cells. However, the method for blast calculation in erythroid-predominant myelodysplastic syndrome (erythroblasts $\geq 50 \%$ ) is not specified either in the FAB or WHO classifications. We retrieved the files of 74 erythroid-predominant myelodysplastic syndrome patients (17\% of all myelodysplastic syndrome) and 192 myelodysplastic syndrome controls (erythroblasts $<\mathbf{5 0} \%$ ). In erythroid-predominant myelodysplastic syndrome, by enumerating blasts from marrow nonerythroid cells rather than from total nucleated cells, 41 of 74 $(55 \%)$ cases would be upgraded, either by disease subcategory or International Prognostic Scoring System. Importantly, the patients with $<5 \%$ blasts demonstrated a superior survival to patients with $\geq \mathbf{5} \%$ blasts $(P=0.002)$; this distinction was lost when blasts were calculated from total-nucleated cells. Of cases with $\geq 5 \%$ blasts, cytogenetics rather than blast count correlated with survival. We conclude that in erythroid-predominant myelodysplastic syndrome, blast calculation as a proportion of marrow nonerythroid rather than total nucleated cells can better stratify patients into prognostically relevant groups.
\end{abstract}

Modern Pathology (2008) 21, 1394-1402; doi:10.1038/modpathol.2008.142; published online 12 September 2008

Keywords: myelodysplastic syndrome; erythroid precursors; blasts enumeration; prognosis; survival

The original 1976 FAB (French-American-British) classification ${ }^{1}$ defined erythroleukemia based on the percentage of myeloblasts in total marrow cells. However, because of the substantial erythroid component present in this disease, it became clear that a diagnosis of acute erythroleukemia with at least $30 \%$ myeloblasts of the total bone marrow elements was difficult to establish. The revised FAB classification ${ }^{2}$ proposed that if $\geq 50 \%$ of nucleated bone marrow cells were erythroblasts, the differentiation between acute erythroleukemia (erythroid/myeloid type, M6a) and myelodysplastic syndrome should be made by assessing the

Correspondence: Dr SA Wang, MD, Department of Hematopathology, 1515 Holcombe Boulevard, Unit 72, Houston, Texas 77030-4009, USA.

E-mail: swang5@mdanderson.org

Received 10 March 2008; revised 15 May 2008; accepted 12 June 2008; published online 12 September 2008 percentage of blasts within the nonerythroid cells, ie, by excluding the erythroblasts from the count. Therefore, a diagnosis of M6a would require $\geq 30 \%$ blasts in the nonerythroid cells; if $<30 \%$, the diagnosis would be myelodysplastic syndrome. However, it was not clearly stated in that classification whether the percentage of blasts should be calculated as a percentage of all nucleated cells or nonerythroid cells to subcategorize myelodysplastic syndrome cases with $\geq 50 \%$ erythroblasts.

In the WHO (World Heath Organization) classification (2001), the blast percentage required to diagnose an acute myeloid leukemia was lowered from $30 \%$ to $20 \%,{ }^{3}$ abolishing the category of refractory anemia with excess blasts in transformation. Accordingly, acute erythroleukemia was also redefined as the presence of $\geq 50 \%$ erythroblasts with myeloblasts $\geq 20 \%$ of nonerythroid cells. However, again it was not specified how the blast 
percentage should be calculated in erythroid-predominant myelodysplastic syndrome which did not fulfill the criteria for acute erythroleukemia. Many hematopathologists enumerate blasts as a proportion of total bone marrow nucleated cells in myelodysplastic syndrome cases irrespective of the erythroid percentage, whereas some others enumerate blasts from bone marrow nonerythroid cells following the criteria established for acute erythroleukemia. The latter method of blast enumeration was briefly mentioned in the report of the response criteria standardization for myelodysplastic syndrome by an international working group but has not been adopted in general practice. ${ }^{4}$

In recent years, many new therapies have been developed to treat myelodysplastic syndrome patients, particularly those with high-risk disease. These new approaches go beyond supportive care and can change the natural course of the disease. ${ }^{5-13}$ However, as the treatments can carry substantial risks, the choice of treatment should ideally be adapted to the risk profile of the patient's disease. It is well-known that in myelodysplastic syndrome patients, the number of bone marrow blasts correlates with the aggressiveness of the disease and patients' overall survival. ${ }^{2,14}$ The range of bone marrow blasts $(<5,5-10$ and $11-20 \%)$ has been included as an important risk factor for International Prognostic Scoring System calculation. ${ }^{15}$ The importance of blast number in myelodysplastic syndrome is also emphasized in disease entity definition by the WHO classification, where refractory anemia with excess blasts is further divided into refractory anemia with excess blasts-1 (5-9\% blasts) and refractory anemia with excess blasts-2 (10-19\% blasts). This WHO subcategorization of myelodysplastic syndrome has been validated in risk stratification by outcome studies. ${ }^{16,17}$

In erythroid-predominant myelodysplastic syndrome, the method chosen for blast enumeration would affect the disease risk categorization, either defined by disease entity or International Prognostic Scoring System group. For example, a case with $3 \%$ blasts and $50 \%$ erythroblasts would be classified as refractory anemia or refractory cytopenia with multilineage dysplasia when the blasts are counted from the total marrow nucleated cells, but would be considered as refractory anemia with excess blasts- 1 when the blasts are calculated from nonerythroid cells. Therefore, we conducted the current study to determine if enumerating blasts from the nonerythroid cells is more effective than enumerating blasts from total bone marrow cells in the prognostic stratification in erythroid-predominant myelodysplastic syndrome patients. The clinicopathological features, disease categorization and survivals were studied and compared to cases of myelodysplastic syndrome without erythroid hyperplasia.

\section{Materials and methods}

\section{Patients}

We retrieved the clinical and laboratory data on all myelodysplastic syndrome patients with erythroblasts $\geq 50 \%$ of total marrow nucleated cells diagnosed at University of Massachusetts Memorial Medical Center and Massachusetts General Hospital over a 7-year period (2000-2007) with institutional review board approval. A group of myelodysplastic syndrome cases with erythroblasts $<50 \%$ in the same time period diagnosed at University of Massachusetts Memorial Medical Center were also included as the control group. The cases lacking adequate bone marrow smears were excluded. In some patients, the disease classification was initially based on FAB criteria $^{18}$ then reclassified according to the WHO proposals, ${ }^{3}$ based upon morphology, laboratory characteristics, cytogenetics and clinical follow-up. Patients with myeloproliferative diseases, chronic myelomonocytic leukemia, atypical chronic myelogenous leukemia, myelodysplastic syndrome/myeloproliferative disease-unclassifiable or cases with myeloblasts $\geq 20 \%$ of total marrow nucleated cells were all excluded. The prognostic score in all of the patients was calculated utilizing the International Prognostic Scoring System. ${ }^{15}$

\section{Morphologic Analysis}

All patients included in the final analysis had representative bone marrow trephine biopsies and smears readily available for evaluation. The Perls reaction for iron was performed on a bone marrow aspirate. Silver impregnation stain for reticulin was performed on biopsy samples when fibrosis was suspected based on Hemotoxylin and Eosin (H\&E) slides. The overall cellularity was estimated on bone marrow biopsies. For the diagnosis of morphologic dysplasia in a bone marrow, features of dyserythropoiesis, dysgranulopoiesis and dysmegakaryopoiesis had to be present in at least $10 \%$ cells of the respective lineage. Unilineage dysplasia was defined by dysplasia involving at least $10 \%$ of cells of a single cell lineage, whereas multilineage dysplasia involved at least $10 \%$ of cells of two or more lineages. The bone marrows were evaluated by two hematopathogists who performed a 500-cell count from multiple fields of the smears on each case and the final blast and erythroid percentages represented an average of these counts. In order to compare our study to data in the literature, lymphocytes and plasma cells were not excluded from the total cell count, following the blast counting method recommended for myelodysplastic syndrome and acute erythroleukemia by FAB classification criteria. In most cases, the blast and erythroid percentages generated by two pathologists were similar. In discrepant cases or cases with $>80 \%$ erythroid 
cells, a third hematopathogist performed an additional 500 count and the average of the three counts was used.

\section{Cytogenetic Analysis}

Fixed preparations obtained from cultured (24 and $48 \mathrm{~h}$ ) bone marrow samples were G-banded and at least 20 or all available (if $<20$ ) metaphase spreads were examined. The criteria defined by the International System for Human Cytogenetic Nomenclature were used for identification of abnormal clones. ${ }^{19}$

\section{Statistical Analysis}

Mann-Whitney test was used for numerical comparison between two groups. Fisher's exact test and $\chi^{2}$-test were applied for categorical variables. Patient survival was estimated by the Kaplan-Meier method from the date of bone marrow diagnosis until death from any cause or until the last patient follow-up. Survival curves were statistically compared by the log-rank test. Differences between two groups were considered statistically significant if $P$-values were less than 0.05 in a two-tailed test.

\section{Results}

\section{MDS with Erythroblasts $\geq \mathbf{5 0} \%$ of Total Marrow Nucleated Cells: Incidence, Bone Marrow Findings and Clinicopathological Features}

A total of 103 consecutive myelodysplastic syndrome patients with erythroblasts $\geq 50 \%$ of bone marrow nucleated cells were identified. A total of 29 cases $(28 \%)$ fulfilled WHO criteria for acute erythroleukemia, and were excluded from the final clinicopathological and outcome comparison. The remaining cases were named as erythroid-predominant myelodysplastic syndrome and comprised $17 \%$ of all myelodysplastic syndrome cases diagnosed at University of Massachusetts Memorial Medical Center and Massachusetts General Hospital between year 2000 and 2007. A control group of 192 consecutive myelodysplastic syndrome patients diagnosed at University of Massachusetts Memorial Medical Center with $<50 \%$ erythroblasts in the bone marrow were similarly analyzed.

As compared to the control group, the erythroidpredominant myelodysplastic syndrome patients were younger $(P=0.03)$ but showed a similar male:female ratio and comparable degree of cytopenias (Table 1). The hematological indices, including hemglobulin, absolute neutrophil count and platelets (complete blood cell count available in 64 of 74 erythroid-predominant myelodysplastic syndrome and 186 of 192 control patients) showed no statistical difference (Table 1). The serum erythropoietin levels were available in 54 of 74 erythroidpredominant myelodysplastic syndrome and 164 of
192 control group patients, and showed no significant difference between two groups $(P=0.27)$. Bone marrow biopsies revealed a comparable cellularity between these two groups $(P=0.2)$. The control group patients had a higher percentage of bone marrow myeloblasts (mean $4.7 \%$, ranging 0-19\%) than erythroid-predominant myelodysplastic syndrome patients (mean $2.9 \%$, range $0-8 \%$ ) when the myeloblasts were enumerated from the total marrow nucleated cells $(P=0.002)$ (Figure 1a). The percentage of bone marrow erythroblasts in erythroidpredominant myelodysplastic syndrome and control cases is shown in Figure 1b.

Karyotypic abnormalities were detected in 43 of $69(62 \%)$ of erythroid-predominant myelodysplastic syndrome patients, compared to 91 of $183(50 \%)$ of the control group. Although this frequency did not reach statistical significance $(P=0.089)$, the erythroid-predominant myelodysplastic syndrome patients showed a higher risk cytogenetic categorization according to International Prognostic Scoring System criteria, with more cases falling into the poor and intermediate risk categories $(P=0.008)$.
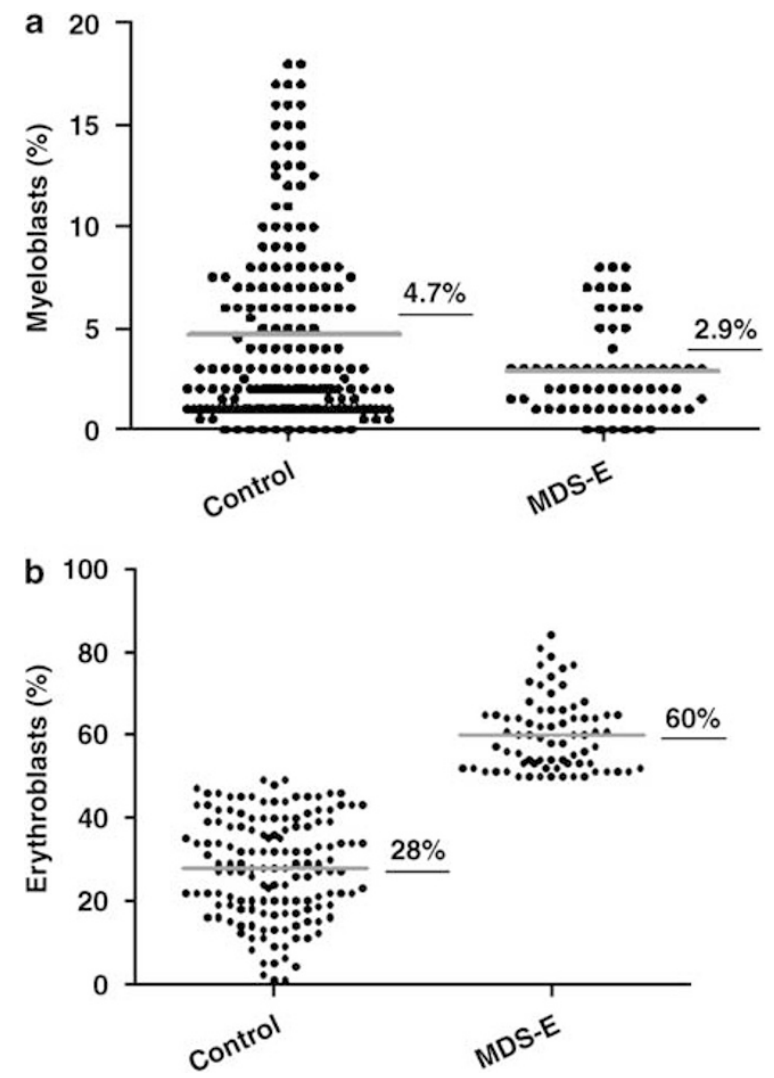

Figure 1 Distribution of myeloblast percentage in erythroidpredominant myelodysplastic syndrome and control cases. (a) Myeloblasts calculated as a proportion of the total marrow nucleated cells $(P=0.002)$; $(b)$ erythroid precursors as a proportion of the total marrow nucleated cells in erythroid-predominant myelodysplastic syndrome versus control myelodysplastic syndrome $(<50 \%$ erythroblasts $)$ cases $(P<0.0001)$. 
Subcategorization of MDS-E: Enumerating Blasts from Total Marrow Nucleated Cells Versus from Marrow Nonerythroid Cells

The WHO classification of the erythroid-predominant myelodysplastic syndrome (using both the methods of blast enumeration) and control myelodysplastic syndrome cases is listed in Table 2. Of 74 erythroid-predominant myelodysplastic syndrome cases, $10(14 \%)$ were therapy-related, a frequency comparable to the control myelodysplastic syndrome cases (21 of $192,11 \%, P=0.27$ ). A significantly higher number of cases with ringed sideroblasts (refractory anemia with ringed sideroblasts and refractory cytopenia with multilineage dysplasia with ringed sideroblasts) were seen in the erythroidpredominant myelodysplastic syndrome group than the control group (33\% versus $12 \%, P=0.006$ ).

Among the erythroid-predominant myelodysplastic syndrome patients, cases with $10-19 \%$ total bone marrow blasts had fulfilled the current WHO criteria of acute erythroleukemia and had been excluded from this study. Therefore, none of the cases in this group can be classified as refractory anemia with excess blasts-2 if blasts are enumerated from the total bone marrow nucleated cells. When the blasts were enumerated from bone marrow nonerythroid cells, 26 of $74(35 \%)$ erythroid-predominant myelodysplastic syndrome patients with $<5 \%$ blasts of the total bone marrow nucleated cells (15 of 26 refractory cytopenia with multilineage dysplasia, and 8 of 14 refractory cytopenia with multilineage dysplasia with ringed sideroblasts) were upgraded to refractory anemia with excess blasts-1 or refractory anemia with excess blasts-2. In contrast, none of the refractory anemia or myelodysplastic syndrome with del(5q) cases were upgraded. Only 1 of 7 primary refractory anemia with ringed sideroblasts was upgraded (Table 1). All 15 cases with 5-9\% marrow blasts (refractory anemia with excess blasts1) were upgraded to refractory anemia with excess blasts-2. In total, 9 of $10(90 \%)$ therapy-related myelodysplastic syndrome cases and 32 of 64 (50\%) primary myelodysplastic syndrome cases were upgraded by enumerating blasts from the marrow nonerythroid cells as compared to from total bone marrow nucleated cells. Accordingly, the International Prognostic Scoring Scoring System scores were also upgraded in all of these patients, resulting in a higher International Prognostic Scoring System category in 35 of 74 patients $(47 \%)$.

When we subcategorized the myelodysplastic syndrome cases by blast percentage $(<5,5-9$ and 10-19\%), erythroid-predominant myelodysplastic syndrome showed a disease category distribution resembling the control group myelodysplastic syndrome cases only when the blasts were calculated from the nonerythroid marrow cells $(P=0.06)$ (Table 2). This subcategorization was dramatically different between the erythroid-predominant myelodysplastic syndrome and control groups when the blasts were calculated from the total bone marrow nucleated cells $(P<0.0001)$, particularly reflecting as the absence of refractory anemia with excess blasts-2 from the erythroid-predominant myelodysplastic syndrome group. Similarly, the International Prognostic Scoring System high-risk group (11-20\% blasts) was eliminated.

\section{Overall Survival Comparison}

Although the blasts as a percentage of marrow total nucleated cells was lower in the erythroid-predominant myelodysplastic syndrome group than the

Table 1 Clinicopathologic features of erythroid-predominant myelodysplastic syndrome ( $\geq 50 \%$ erythroblasts), in comparison with erythroblasts $<50 \%$ control group

Erythroid-predominant myelodysplastic Controls (erythroid $\quad \mathrm{P}$ syndrome (erythroid precursors $\geq 50 \%$ ) precursors $<50 \%$ )

\begin{tabular}{|c|c|c|c|}
\hline & $\begin{array}{l}\text { Erythroid-predominant myelodysplastic } \\
\text { syndrome (erythroid precursors } \geq 50 \% \text { ) }\end{array}$ & $\begin{array}{l}\text { Controls (erythroid } \\
\text { precursors }<50 \% \text { ) }\end{array}$ & $\mathrm{P}$ \\
\hline Patients $(n)$ & 74 & 192 & \\
\hline Age (mean, range, years) & $67(35-87)$ & $70(26-91)$ & $P=0.03$ \\
\hline Male:female (M:F) & 49:25 & 127:65 & $P=1.0$ \\
\hline Hemoglobin (Hb) (g per $100 \mathrm{ml}$ ) & $10.0 \pm 0.2(4.5-14.1)$ & $10.3 \pm 0.1(6.4-14.2)$ & $P=0.17$ \\
\hline White blood count (WBC) $\left(\times 10^{9}\right.$ per 1$)$ & $4.5 \pm 0.4(1.0-14.9)$ & $4.5 \pm 0.2(0.3-19)$ & $P=0.90$ \\
\hline Absolute neutrophil count $(\mathrm{ANC})\left(\times 10^{9}\right.$ per 1$)$ & $2.0 \pm 0.2(0.3-10.2)$ & $2.6 \pm 0.2(0.1-14.5)$ & $P=0.39$ \\
\hline Platelets $\left(\times 10^{9}\right.$ per 1$)$ & $139 \pm 15(15-633)$ & $137 \pm 10(2-799)$ & $P=0.91$ \\
\hline Serum erythropoietin (IU) & $276 \pm 80(7-3048)$ & $404 \pm 57(18-2980)$ & $P=0.27$ \\
\hline \multicolumn{4}{|l|}{ Bone marrow } \\
\hline Cellularity (\%) & $65 \pm 3(5-100)$ & $60 \pm 2(5-100)$ & $P=0.20$ \\
\hline Erythroblasts (\%) & $60(50-84)$ & $28(3-49)$ & $P<0.0001$ \\
\hline Blasts of total nucleated cells (\%) & $2.9 \pm 0.3(0-8)$ & $4.7 \pm 0.3(0-19)$ & $P=0.002$ \\
\hline Cytogenetics (abnormal/total cases) & $43 / 69(62 \%)$ & $91 / 181(50 \%)$ & ${ }^{\mathrm{a}} \mathrm{P} 1=0.089 / \mathrm{P} 2=0.008$ \\
\hline IPSS-good & 30 & 116 & \\
\hline IPSS-intermediate & 16 & 33 & \\
\hline IPSS-poor & 23 & 32 & \\
\hline
\end{tabular}

IPSS: International Prognostic Scoring System.

${ }^{a}$ Cytogenetics (cases with abnormal cytogenetics versus total cases with available cytogenetic information) (P1: comparison of abnormal karyotype frequency; P2: IPSS risk category distribution). 
Table 2 Erythroid-predominant myelodysplastic syndrome with $\geq 50 \%$ erythroblasts in the marrow, disease categories by myeloblasts enumeration from total marrow nucleated cells and of non-erythroid cells, in comparison with erythroblasts $<50 \%$ control group

\begin{tabular}{|c|c|c|c|}
\hline \multirow[t]{2}{*}{ WHO categories } & \multicolumn{2}{|c|}{$\begin{array}{l}\text { Erythroid-predominant myelodysplastic syndrome } \\
\text { (erythroid precursors } \geq 50 \%)(\mathrm{N}=74)\end{array}$} & \multirow[t]{2}{*}{$\begin{array}{c}\text { Controls (erythroid } \\
\text { precursors }<50 \%)(\mathrm{N}=192\end{array}$} \\
\hline & $\begin{array}{l}\text { Blasts calculated from } \\
\text { total nucleated cells }\end{array}$ & $\begin{array}{l}\text { Blasts calculated from } \\
\text { nonerythroid cells }\end{array}$ & \\
\hline Therapy-related MDS & $10(14 \%)$ & & $21(11 \%)$ \\
\hline RA & 0 & 0 & 3 \\
\hline RARS & 1 & 0 & 2 \\
\hline RCMD-RS & 2 & 0 & 0 \\
\hline RCMD & 6 & 1 & 8 \\
\hline RAEB-1 & 1 & 5 & 6 \\
\hline RAEB-2 & 0 & 4 & 2 \\
\hline Primary MDS & $64(86 \%)$ & & $171(89 \%)$ \\
\hline $5 q-$ & $1(2 \%)$ & $1(2 \%)$ & $4(2 \%)$ \\
\hline RA & $5(8 \%)$ & $5(8 \%)$ & $17(10 \%)$ \\
\hline RARS & $7(11 \%)$ & $6(9 \%)$ & $7(4 \%)$ \\
\hline RCMD & $20(31 \%)$ & $10(16 \%)$ & $49(29 \%)$ \\
\hline RCMD-RS & $14(22 \%)$ & $8(13 \%)$ & $14(8 \%)$ \\
\hline MDS-U & $3(5 \%)$ & $2(3 \%)$ & $11(6 \%)$ \\
\hline RAEB-1 & $14(22 \%)$ & $15(23.4 \%)$ & $37(22 \%)$ \\
\hline RAEB-2 & 0 & $17(27 \%)$ & $32(19 \%)$ \\
\hline \multicolumn{4}{|l|}{ Blast ranges } \\
\hline$<5 \%$ & 59 & 33 & 115 \\
\hline $5-9 \%$ & 15 & 20 & 43 \\
\hline $10-19 \%$ & $\mathrm{NA}^{\mathrm{a}}$ & 21 & 34 \\
\hline \multicolumn{4}{|c|}{ IPSS-number of patients and their median survival (months) } \\
\hline Low & $22(73)$ & $14(73)$ & 73 (not reached) \\
\hline Int-1 & 32 (33) & 24 (33) & $59(36)$ \\
\hline Int-2 & $15(9)$ & $20(14)$ & $31(18)$ \\
\hline High & 0 & $11(9)$ & $17(11)$ \\
\hline
\end{tabular}

RA: refractory anemia; RARS: refractory anemia with ringed sideroblasts; RCMD: refractory cytopenia with multilineage dysplasia; RCMD-RS: refractory cytopenia with multilineage dysplasia and ringed sideroblasts; MDS-U: myelodysplastic syndrome-unclassifiable; RAEB: refractory anemia with excess blasts; IPSS: International Prognostic Scoring System.

${ }^{\mathrm{a}} \mathrm{NA}$ : Not applicable because all cases with $10-19 \%$ blasts in erythroid-predominant myelodysplastic syndrome would meet the criteria for acute erythroleukemia.

control myelodysplastic syndrome group (Figure 1a), the median overall survival in the two groups was comparable (32 versus 31 months, Kaplan-Meier estimate, log-rank: $P=0.52$; Figure 2).

Of the patients with erythroid-predominant myelodysplastic syndrome $(n=74)$, when the blasts were enumerated from the total bone marrow nucleated cells, 59 patients had $<5 \%$ blasts and 15 patients had blasts in the refractory anemia with excess blasts range (5-9\%). There was no overall survival difference between these two groups of patients (Figure 3a), irrespective of whether the therapy-related myelodysplastic syndrome cases were included (log-rank, $P=0.47$ ) or excluded (logrank, $P=0.41$ ) (figure not shown) from the analysis. In contrast, when the blasts were enumerated from the marrow nonerythroid cells, patients with $<5 \%$ blasts $(n=33)$ had a superior overall survival to patients with blasts in the range of refractory anemia with excess blasts $(n=41)$ (log-rank, $P=0.0024)$ (Figure $3 \mathrm{~b}$ ). This significance in overall survival was maintained when therapy-related myelodysplastic



Figure 2 The erythroid-predominant myelodysplastic syndrome patients showed a comparable overall survival to control group patients $(<50 \%$ erythroblasts $)(P=0.52)$, despite a lower blast number as a proportion of total marrow nucleated cells in erythroid-predominant myelodysplastic syndrome.

syndrome were excluded from the comparison (logrank, $P=0.02$ ).

We compared the erythroid-predominant myelodysplastic syndrome cases with $<5 \%$ blasts defined 

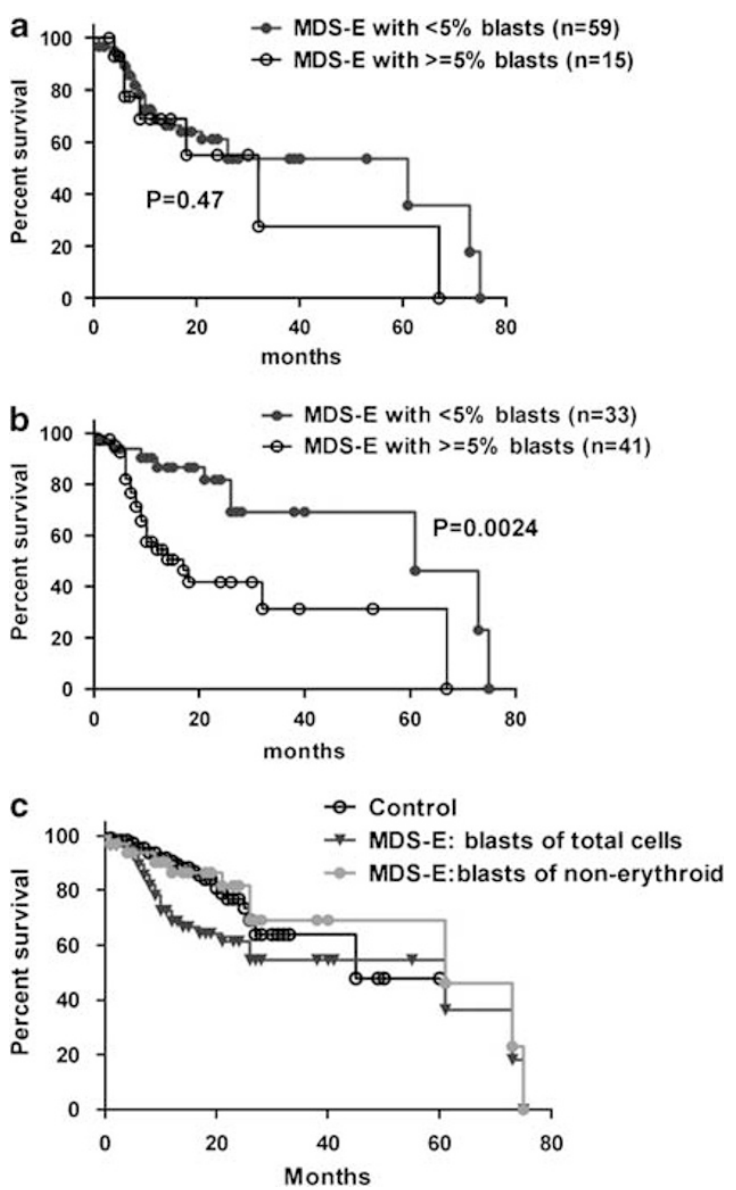

Figure 3 Overall survival comparison in erythroid-predominant myelodysplastic syndrome: (a) When the blasts were enumerated as a proportion of the total marrow nucleated cells, cases with $<5 \%$ blasts showed no difference in overall survival to cases with $\geq 5 \%$ blasts (refractory anemia with excess blasts) $(P=0.47)$; (b) when the blasts were enumerated as a proportion of marrow nonerythroid cells, cases with $<5 \%$ blasts showed a superior overall survival to refractory anemia with excess blasts $(P=0.0024) ; \quad(\mathbf{c})$ erythroid-predominant myelodysplastic syndrome cases with $<5 \%$ blasts defined by blast calculation from nonerythroid cells showed a similar overall survival to the control myelodysplastic syndrome cases with $<5 \%$ blasts but a significantly inferior survival when blasts were calculated from total marrow nucleated cells $(P=0.03)$.

by blast enumeration from the total bone marrow nucleated cells $(n=59)$ or from the nonerythroid cells $(n=33)$ to the control group cases with $<5 \%$ blasts $(n=115)$. When $<5 \%$ blasts was determined by blast enumeration of total marrow nucleated cells, the overall survival of the erythroid-predominant myelodysplastic syndrome patients with $<5 \%$ blasts was significantly inferior to the control group patients with $<5 \%$ blasts (Figure 3c) (log-rank, $P=0.03$ ). In contrast, if the blast count was determined by enumeration of the blast in nonerythroid cells, the overall survival of the erythroidpredominant myelodysplastic syndrome patients with $<5 \%$ blasts was similar to the control group patients with $<5 \%$ blasts (log-rank, $P=0.68$ ) (Figure 3c).
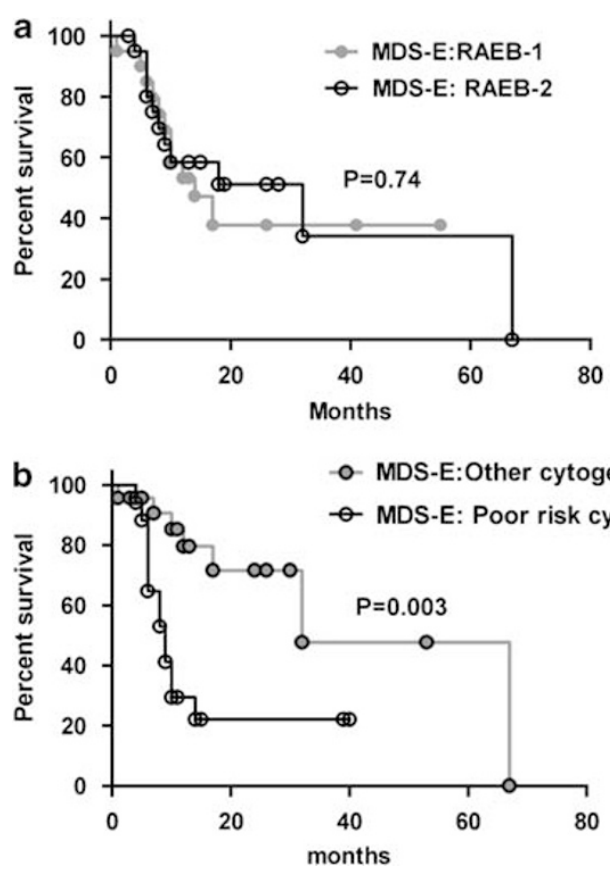

Figure 4 Erythroid predominant myelodysplastic syndrome with $\geq 5 \%$ blasts defined by blast calculation from marrow nonerythroid cells. (a) There was no survival difference between refractory refractory anemia with excess blasts-1 and refractory anemia with excess blasts-2 groups $(P=0.74)$. (b) Patients with a high-risk karyotype International Prognostic Scoring System Score showed inferior survival to patients with a normal karyotype or low- or intermediate-risk karyotypic abnormality $(P=0.003)$

We also compared the cases with blasts in the range of refractory anemia with excess blasts. If the blasts were enumerated from the total nucleated cells, 15 patients would be classified as refractory anemia with excess blasts-1 and none would be refractory anemia with excess blasts-2. In contrast, when the blasts were calculated from nonerythroid cells, 41 patients would be classified as refractory anemia with excess blasts (20 refractory anemia with excess blasts-1 and 21 refractory anemia with excess blasts-2). There was no difference in overall survival found between the refractory anemia with excess blasts-1 and refractory anemia with excess blasts-2 patients, including or excluding therapy-related myelodysplastic syndrome $(P=0.74$ and 0.88 respectively) (Figure 4a). However, refractory anemia with excess blasts patients (as defined by blast enumeration from nonerythroid cells) with a karyotype in the poor risk International Prognostic Scoring System category showed an inferior overall survival to patients with a good or intermediate risk karyotype, whether including $(P=0.003)$ (Figure $4 \mathrm{~b})$ or excluding $(P=0.03)$ therapy-related myelodysplastic syndrome (figure not shown).

The overall survival comparison by International Prognostic Scoring System groups is shown in Table 2. When the blasts were calculated from the nonerythroid cells, the overall survival was statisti- 
cally significant among all four groups (Low, Int-1, Int-2 and High) $(P=0.02)$. When blasts were calculated from the total marrow nucleated cells, although three of the groups (Low, Int-1 and Int-2) also demonstrated overall survival difference $(P=0.006)$, none of the cases would be classified in the High group, thus eliminating this group from the erythroid-predominant myelodysplastic syndrome patients.

\section{Discussion}

In this study, we showed that myelodysplastic syndrome with erythroid predominance $(\geq 50 \%$ erythroblasts) comprises a small but significant proportion of myelodysplastic syndrome cases. We compared two methods of enumerating blasts in this group (as a percentage of total bone marrow cells or nonerythroid cells) to classify this group of cases. Although the number of bone marrow blasts of the erythroid-predominant myelodysplastic syndrome patients was lower than in a control group of myelodysplastic syndrome lacking erythroid predominance, the patients with erythroid-predominant myelodysplastic syndrome did not show a superior overall survival. Blast enumeration as a proportion of total marrow nucleated cells lost its prognostic power in this group of patients. Instead, blast calculation as a percentage of the marrow nonerythroid cells resulted in a more relevant disease subcategorization based on outcome studies.

We have shown that in the erythroid-predominant myelodysplastic syndrome patients, enumerating the blasts from the total marrow nucleated cells failed to prognostically separate the patients with $<5 \%$ blasts (presumptively low grade) from the cases of refractory anemia with excess blasts (high grade) by outcome study. In contrast, by enumerating the blasts of marrow nonerythroid cells, a $<5 \%$ blast cutoff identified a subset of patients who had a relatively indolent clinical course. This conclusion held true whether therapy-related myelodysplastic syndrome cases were included or excluded. In 2006, Mazzella et $a l^{20}$ studied a group of patients with 'clinical impression of myelodysplastic syndrome, preleukemia or leukemia' and erythroid predominance. In their study, they calculated the myeloblasts as a percentage of nonerythroid cells. Although in their series the diseases were not clearly categorized and there was no comparison to a control group of patients, they showed that the patients' prognosis could be stratified by blast ranges, showing a superior overall survival in patients with $<5 \%$ blasts to patients with $\geq 5 \%$ blasts, as well as a superior overall survival with $5-9 \%$ blasts as compared to $10-19 \%$. In our study, we were able to show that refractory anemia with excess blasts group defined by blast enumeration of marrow nonerythroid cells had a similar overall survival to the control group refractory anemia with excess blasts with $<50 \%$ erythroblasts. However, the overall survival in patients with erythroidpredominant refractory anemia with excess blasts could not be further stratified by the blast range (5-9 and 10-19\%) alone. Instead, a high-risk karyotype defined by International Prognostic Scoring System criteria predicted a very poor prognosis in this group of patients irrespective of the blast count.

Significantly, we have demonstrated that although some erythroid-predominant myelodysplastic syndrome cases (such as refractory anemia, and 5q-syndrome and majority primary refractory anemia with ringed sideroblasts which were not upgraded by enumerating the blasts as a percentage of the nonerythroid cells) appear to be truly low grade, a significant number of such cases are clinically aggressive, despite a lower overall number of myeloblasts in the bone marrow. Enumerating blasts as a percentage of the nonerythroid cells identified this group of patients with more aggressive behavior, whereas this group would be lost among refractory cytopenia with multilineage dysplasia and refractory anemia cases (with $<5 \%$ blasts) if blasts were enumerated as a percentage of total bone marrow cells. Because counting blasts as a percentage of nonerythroid cells amplifies small differences in blast percentages, a large number of total cells (at least 500 cells, as recommended by the WHO) ${ }^{3}$ should be counted to ensure precision, particularly when the number of nonerythroid cells is relatively small.

The biology of the erythroid proliferation in myelodysplastic syndrome is not well understood; however, the mechanisms of erythroid proliferation may differ between refractory anemia with ringed sideroblasts and other erythroid-predominant myelodysplastic syndrome cases as they demonstrate different gene expression profiling. ${ }^{21}$ In our study, refractory anemia with excess blasts with erythroid predominance comprised a significantly higher proportion of cases with a poor-risk karyotype, such as $-7,-7 q,-5$, or a complex karyotype, compared to the control group, and these cases showed a poor overall survival irrespective of the blast ranges. We further demonstrated overall survival difference by International Prognostic Scoring System risk groups in these patients, where cytogenetic risk is incorporated into the scoring system. It has been acknowledged that there is a great deal of clinical, morphologic, and cytogenetic overlap between refractory anemia with excess blasts and acute erythroleukemia. ${ }^{22,23}$ In a study conducted by Olopade et $a l,{ }^{24}$ once a patient fulfilled the FAB criteria for M6a, the percentage of blast cells did not correlate with survival in the subset of patients with chromosomal abnormalities of 5 and/or 7. Our findings in refractory anemia with excess blasts further affirm the clinical and biological similarity between erythroid-predominant refractory anemia with excess blasts and acute erythroleukemia. 
These findings also suggest that certain karyotypic alternations in erythroid-predominant myelodysplastic syndrome dictate its biological aggressiveness. Microarray-based gene expression profiling studies $^{25-27}$ have shown potential in defining specific and prognostically relevant gene signatures in myelodysplastic syndrome, which may enhance our understanding of the biology and clinical behavior of this disease.

In addition to the overall survival comparison, erythroid-predominant myelodysplastic syndrome showed a more comparable diagnostic categorization to the control myelodysplastic syndrome cases if the blasts were enumerated from the marrow nonerythroid cells (Table 2). The International Prognostic Scoring System would include all risk groups, including the high-risk group, and the median overall survival of each group would be more comparable to that reported in literature. ${ }^{15}$ Interestingly, 9 out of 10 (90\%) therapy-related myelodysplastic syndrome would be upgraded from $<5 \%$ blasts to refractory anemia with excess blasts, better reflecting the aggressiveness of therapy-related myelodysplastic syndrome. In contrast, the myelodysplastic syndrome subcategorization would be dramatically different from the control group if the blasts were calculated from the total bone marrow nucleated cells, mainly due to the absence of refractory anemia with excess blasts- 2 and of cases falling into the International Prognostic Scoring System high-risk category. This further suggests that erythroid-predominant refractory anemia with excess blasts forms a continuum with acute erythroleukemia and should similarly be defined by blast calculation from the marrow nonerythroid cells, a method that has shown its practical validity in acute erythroleukemia cases. ${ }^{20,24,28}$

In summary, we have shown that cases of myelodysplastic syndrome with erythroid predominance may behave in an aggressive fashion even when the myeloblasts account for a relatively small proportion $(<5 \%)$ of the total marrow elements. Conventional counting of blasts as a percentage of the total nucleated bone marrow cells fails to accurately subcategorize this group of myelodysplastic syndrome and stratify the patients into appropriate risk groups. Instead, our data support the enumeration of blasts as a proportion of bone marrow nonerythroid cells for both erythroid-predominant myelodysplastic syndrome and acute erythroleukemia cases.

\section{Disclosure/conflict of interest}

The authors have no conflict of interest to declare. The views expressed in this article are those of the authors and do not reflect the official policy of the Department of Defense or other Departments of the United States Government.

\section{References}

1 Bennett JM, Catovsky D, Daniel MT, et al. Proposals for the classification of the acute leukaemias. FrenchAmerican-British (FAB) co-operative group. $\mathrm{Br}$ J Haematol 1976;33:451-458.

2 Bennett JM, Catovsky D, Daniel MT, et al. Proposed revised criteria for the classification of acute myeloid leukemia. A report of the French-American-British Cooperative Group. Ann Intern Med 1985;103: 620-625.

3 Harris NL, Jaffe ES, Diebold J, et al. World Health Organization classification of neoplastic diseases of the hematopoietic and lymphoid tissues: report of the Clinical Advisory Committee meeting-Airlie House, Virginia, November 1997. J Clin Oncol 1999;17: 3835-3849.

4 Cheson BD, Bennett JM, Kantarjian H, et al. Report of an international working group to standardize response criteria for myelodysplastic syndromes. Blood 2000;96:3671-3674.

5 de Witte T, Brand R, van Biezen A, et al. The role of stem cell source in autologous hematopoietic stem cell transplantation for patients with myelodysplastic syndromes. Haematologica 2006;91: $750-756$.

6 Cutler CS, Lee SJ, Greenberg P, et al. A decision analysis of allogeneic bone marrow transplantation for the myelodysplastic syndromes: delayed transplantation for low-risk myelodysplasia is associated with improved outcome. Blood 2004;104:579-585.

7 Santini V, Gozzini A, Bosi A. Treatment options in myelodysplastic syndromes: a new frontier. J Chemother 2008;20:291-296.

8 Nakai K, Kanda Y, Fukuhara S, et al. Value of chemotherapy before allogeneic hematopoietic stem cell transplantation from an HLA-identical sibling donor for myelodysplastic syndrome. Leukemia 2005;19:396-401.

9 Kantarjian H, Issa JP, Rosenfeld CS, et al. Decitabine improves patient outcomes in myelodysplastic syndromes: results of a phase III randomized study. Cancer 2006;106:1794-1803.

10 Kuendgen A, Schmid M, Schlenk R, et al. The histone deacetylase (HDAC) inhibitor valproic acid as monotherapy or in combination with all-trans retinoic acid in patients with acute myeloid leukemia. Cancer 2006;106:112-119.

11 Kuendgen A, Strupp C, Aivado M, et al. Treatment of myelodysplastic syndromes with valproic acid alone or in combination with all-trans retinoic acid. Blood 2004;104:1266-1269.

12 Fenaux P, Raza A, Mufti GJ, et al. A multicenter phase 2 study of the farnesyltransferase inhibitor tipifarnib in intermediate- to high-risk myelodysplastic syndrome. Blood 2007;109:4158-4163.

13 Valent P, Horny HP, Bennett JM, et al. Definitions and standards in the diagnosis and treatment of the myelodysplastic syndromes: consensus statements and report from a working conference. Leuk Res 2007;31:727-736.

14 Bennett JM. A comparative review of classification systems in myelodysplastic syndromes (MDS). Semin Oncol 2005;32:S3-S10.

15 Greenberg P, Cox C, LeBeau MM, et al. International scoring system for evaluating prognosis in myelodysplastic syndromes. Blood 1997;89:2079-2088. 
16 Germing U, Gattermann N, Strupp C, et al. Validation of the WHO proposals for a new classification of primary myelodysplastic syndromes: a retrospective analysis of 1600 patients. Leuk Res 2000;24:983-992.

17 Malcovati L, Porta MG, Pascutto C, et al. Prognostic factors and life expectancy in myelodysplastic syndromes classified according to WHO criteria: a basis for clinical decision making. J Clin Oncol 2005;23: 7594-7603.

18 Bennett JM, Catovsky D, Daniel MT, et al. Proposals for the classification of the myelodysplastic syndromes. Br J Haematol 1982;51:189-199.

19 Mitelman F. An International System for Human Cytogenetic Nomenclature (1995), Vol. 1995. Karger: Basel; Farmington, CT, 1995.

20 Mazzella FM, Smith D, Horn P, et al. Prognostic significance of pronormoblasts in erythrocyte predominant myelodysplastic patients. Am J Hematol 2006;81:484-491.

21 Lee YT, Miller LD, Gubin AN, et al. Transcription patterning of uncoupled proliferation and differentiation in myelodysplastic bone marrow with erythroidfocused arrays. Blood 2001;98:1914-1921.

22 Park S, Picard F, Dreyfus F. Erythroleukemia: a need for a new definition. Leukemia 2002;16:1399-1401.
23 Park S, Picard F, Guesnu M, et al. Erythroleukaemia and RAEB-t: a same disease? Leukemia 2004;18: 888-890.

24 Olopade OI, Thangavelu M, Larson RA, et al. Clinical, morphologic, and cytogenetic characteristics of 26 patients with acute erythroblastic leukemia. Blood 1992;80:2873-2882.

25 Hofmann WK, de Vos S, Komor M, et al. Characterization of gene expression of CD34+ cells from normal and myelodysplastic bone marrow. Blood 2002;100:3553-3560.

26 Pellagatti A, Esoof N, Watkins F, et al. Gene expression profiling in the myelodysplastic syndromes using cDNA microarray technology. $\mathrm{Br} \mathrm{J}$ Haematol 2004;125:576-583.

27 Pellagatti A, Cazzola M, Giagounidis AA, et al. Gene expression profiles of CD34+ cells in myelodysplastic syndromes: involvement of interferon-stimulated genes and correlation to FAB subtype and karyotype. Blood 2006;108:337-345.

28 Park S, Picard F, Azgui Z, et al. Erythroleukemia: a comparison between the previous FAB approach and the WHO classification. Leuk Res 2002;26: 423-429. 